\title{
An extreme ultraviolet interferometer using high-order harmonic generation from successive sources
}

\author{
D.E. Laban, N.S. Gaffney, R.P.M.J.W. Notermans, T.T.J. Clevis, W.C. \\ Wallace, A.J. Palmer, M.G. Pullen, D. Kielpinski, and R.T. Sang* \\ $A R C$ Centre of Excellence for Coherent X-Ray Science, \\ Griffith University, Brisbane, Queensland, Australia and \\ Australian Attosecond Science Facility and Centre for Quantum Dynamics, \\ Griffith University, Brisbane, Queensland, Australia \\ D. Jiang and H.M. Quiney \\ ARC Centre of Excellence for Coherent X-Ray Science, \\ University of Melbourne, Melbourne, Victoria, Australia \\ I.V. Litvinyuk \\ Australian Attosecond Science Facility and Centre for Quantum Dynamics, \\ Griffith University, Brisbane, Queensland, Australia
}

\begin{abstract}
Recent experiments utilising extreme ultraviolet (XUV) radiation generated by the high-order harmonic generation (HHG) process have been able to probe electron dynamics at timescales approaching or exceeding that of the Bohr orbit time $(\approx 150$ as $)$. As these experiments become more common, new methods and techniques will be required to deal with the short-wavelengths involved. We present a new interferometer technique whereby multiple XUV is generated at different positions within the laser focus (i.e. from successive sources) with a highly-controllable time delay. The induce time delay is found to be a direct consequence of the Gouy phase shift. Ultimately, the apparatus is capable of accessing unprecedented timescales by allowing delays down to better than 100 zeptoseconds, an impossible feat with conventional techniques.
\end{abstract}

When an atom is exposed to a time varying electric field in which the field strength approaches the Coulomb binding energy of the outer electrons, the atom can undergo tunnel ionisation. The free electron, depending on the time of ionisation, can recombine with its parent ion, resulting in the emission of a photon at a time and energy determined by the trajectory of the electron in the accelerating electric field. This process is called high-order harmonic generation (HHG), with the periodic nature of the driving electric-field giving rise to odd-harmonics. With the development of ultrafast mode-locked lasers, it has been possible to create isolated attosecond pulses by gating the HHG emission to one half-cycle with amplitude via a few-cycle pulse $[1,2]$ or with polarisation and two-colour effects $[7,8]$. Amplitude gating requires spectrally filtering the cut-off region of the HHG spectrum that is generated by a carrier-envelope phase (CEP) locked few-cycle laser [9] with pulses as short as 80 as being created with this method [10]. The duration of these attosecond pulses routinely approaches or exceeds the Bohr orbit time of an electron in a hydrogen atom, $\approx 150$ as, making it possible to directly observe electron motion for the first time. One difficulty in working with short-wavelength HHG radiation is that it is technically challenging to impart small time delays between multiple pulses with current interferometric techniques. The best resolution currently available is 20 attoseconds in a Mach-Zehnder interferometer with a co-propagating $532 \mathrm{~nm}$ reference laser used to provide stabilization feedback [5]. In this work, it is shown how the Gouy phase shift of the driving laser through it's focus can be utilised to precisely alter the electron recombination and photon emission time of the HHG process. This is then used to impart small time delays between XUV radiation emitted by successive sources at different longitudinal positions within the focus of a single driving laser. Previous work with successive sources in a single laser focus demonstrated the possibility for increasing HHG yields, however the physical mechanism responsible for increasing the yield at certain separations was not adequately revealed [6]. The resolution of the time delay that can be introduced with this entirely passive method is found to be better than 100 zeptoseconds.

As a laser passes through a focus, the phase velocity increases such that it undergoes a $\pi$ phase shift over a few Rayleigh ranges, $z_{R}$. This is called the Gouy phase shift [14] and it can be found at different focal positions, $z$, by the following expression,

$$
\phi_{\text {Gouy }}(z)=-\arctan \left(\frac{z}{z_{\mathrm{R}}}\right) .
$$

The Gouy phase shift has been previously experimentally verified in the few-cycle pulse regime to shift the carrierenvelope phase (CEP) [13]. In HHG, the emission of a photon depends on the trajectory that the ionised electron takes in the electric-field of the driving laser. As 

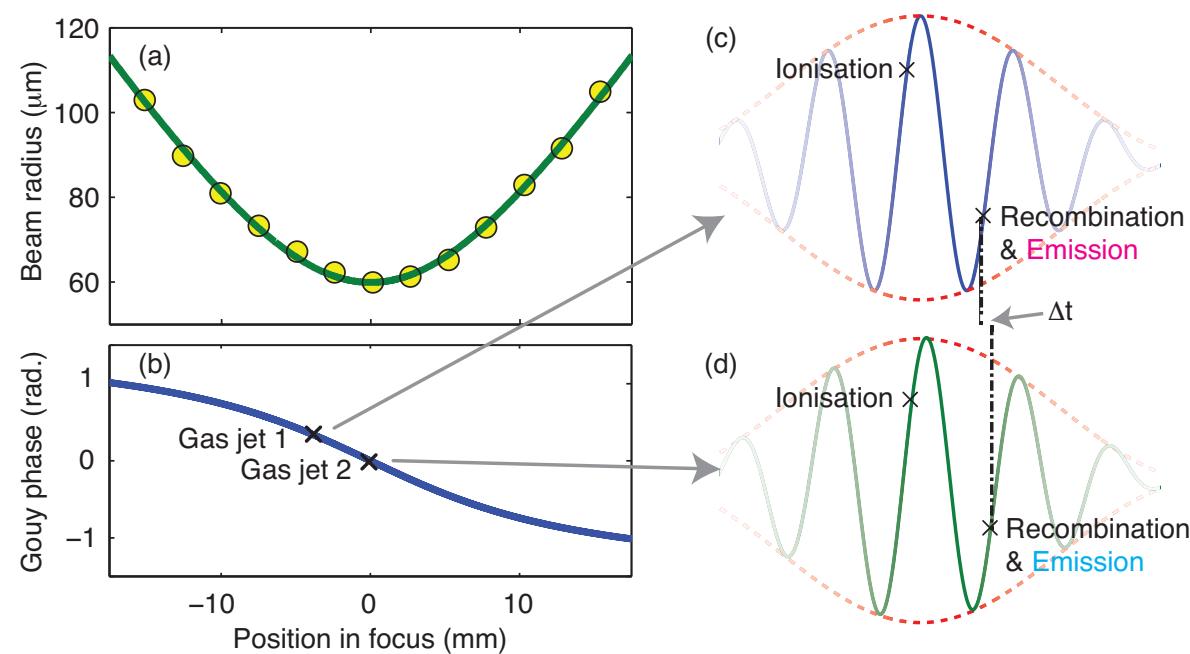

(e)

Two extreme ultraviolet pulses arrive at the detector with a phase offset

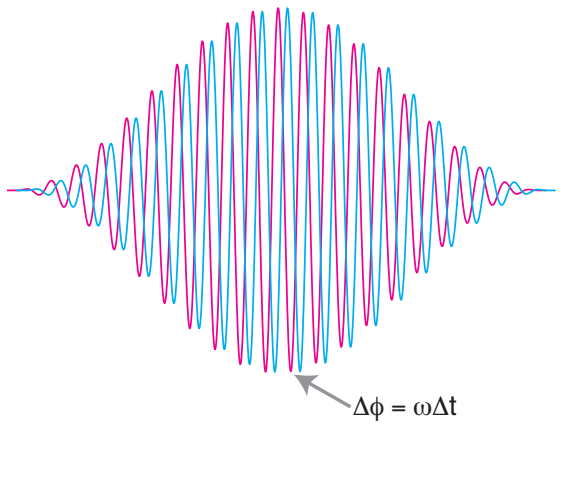

FIG. 1. A schematic depicting the principle of operation of the HHG interferometer. In (a) the beam waist is measured at different positions along the focus (yellow circles) and a fit to these data points (green line) allows the Rayleigh range to be calculated. From the calculated Rayleigh range, the Gouy phase can be calculated at different focal positions (b). By situating the two gas jets at different positions within the focus, the carrier-envelope phase of the driving laser gets shifted between interacting with gas jet 1 (c) and gas jet $2(\mathrm{~d})$. This results in a small delay time $\Delta t$ between the electron recombining. Finally, at the detector (e), two XUV pulses arrive with some phase offset either causing destructive or constructive interference to decrease or increase the total HHG yield, respectively.

such, any delay that is introduced to the driving laser's electric-field will result in a similar delay of the HHG emission. Due to the Gouy phase shift, it is better to measure all ionisation and recombination times relative to the peak of the envelope of the pulse as the group velocity of the driving laser remains unchanged through the focus. An expression for the emission phase for a particular harmonic is given by the Lewenstein model [24, 25],

$$
\phi=q \omega t_{\mathrm{r}}-\frac{1}{\hbar} S\left(p_{\mathrm{st}}, t_{\mathrm{i}}, t_{\mathrm{r}}\right) .
$$

Where $q$ is the harmonic number, $\omega$ is the angular frequency of the driving laser, $t_{\mathrm{r}}$ is the time when the electron recombines with the parent ion to produce a HHG photon, and $S$ is the semi-classical action of the electron in the field which depends on the canonical momentum $p_{\text {st }}$, and the electron ionisation and recombination times $t_{\mathrm{i}}$ and $t_{\mathrm{r}}$. The first term in this expression will be directly influenced by the position of the generating medium in the laser focus as per the Gouy phase relationship,

$$
t_{\mathrm{r}}(z)=t_{\mathrm{r}}^{\prime}+\frac{\phi_{\text {Gouy }}(z)}{\omega} .
$$

Where $t_{\mathrm{r}}^{\prime}$ is the return time for a trajectory measured relative to the electric-field as opposed to the envelope of the pulse. The second term in the emission phase expression is proportional to the the intensity and transit time of the electron in the field (i.e. $S \propto I \times\left[t_{\mathrm{r}}-t_{\mathrm{i}}\right]$ ). It is now possible to write down an expression for the phase difference between two different generating medium located at positions $z_{1}$ and $z_{2}$ within the same laser focus,

$$
\begin{aligned}
\Delta \phi= & q \omega\left(t_{\mathrm{r} 1}-t_{\mathrm{r} 2}\right)-\frac{1}{\hbar}\left(S_{1}-S_{2}\right) \\
= & q\left(\phi_{\text {Gouy }}\left(z_{1}\right)-\phi_{\text {Gouy }}\left(z_{2}\right)\right)+q \omega\left(t_{\mathrm{r} 1}^{\prime}-t_{\mathrm{r} 2}^{\prime}\right) \\
& -\frac{1}{\hbar}\left(S_{1}-S_{2}\right) .
\end{aligned}
$$

The second term in this expression is $\approx 0$ as the trajectory does not differ substantially for a given harmonic. Near the centre of the laser focus, the Gouy phase is changing rapidly with position while the intensity is only slowly decreasing. In the limit of slowly changing intensity the HHG phase difference expression reduces down to the first term only, implying that the delay in the electron recombination time between the two generating media due to the Gouy phase is wholly responsible. A first-order Taylor expansion of Eq. (4) can be used to find an approximate expression for the phase difference in terms of the focus position $z$.

$$
\Delta \phi \approx q \frac{d \phi_{\text {Gouy }}}{d z} \Delta z-\frac{d \phi_{S}}{d z} \Delta z
$$

where the action term has been converted to a phase, $\phi_{S}$, to simplify the expression. Ultimately this expression shows that a small Gouy phase shift in the driving laser can lead to a phase difference between two HHG emissions that is $q$ times larger. This is essentially an interferometer that can introduce a time delay between the creation of two or more HHG emissions depending on the relative positions of the generating media within the 
laser focus. Fig. (1) gives a diagrammatic representation of the operation principles of the HHG interferometer.

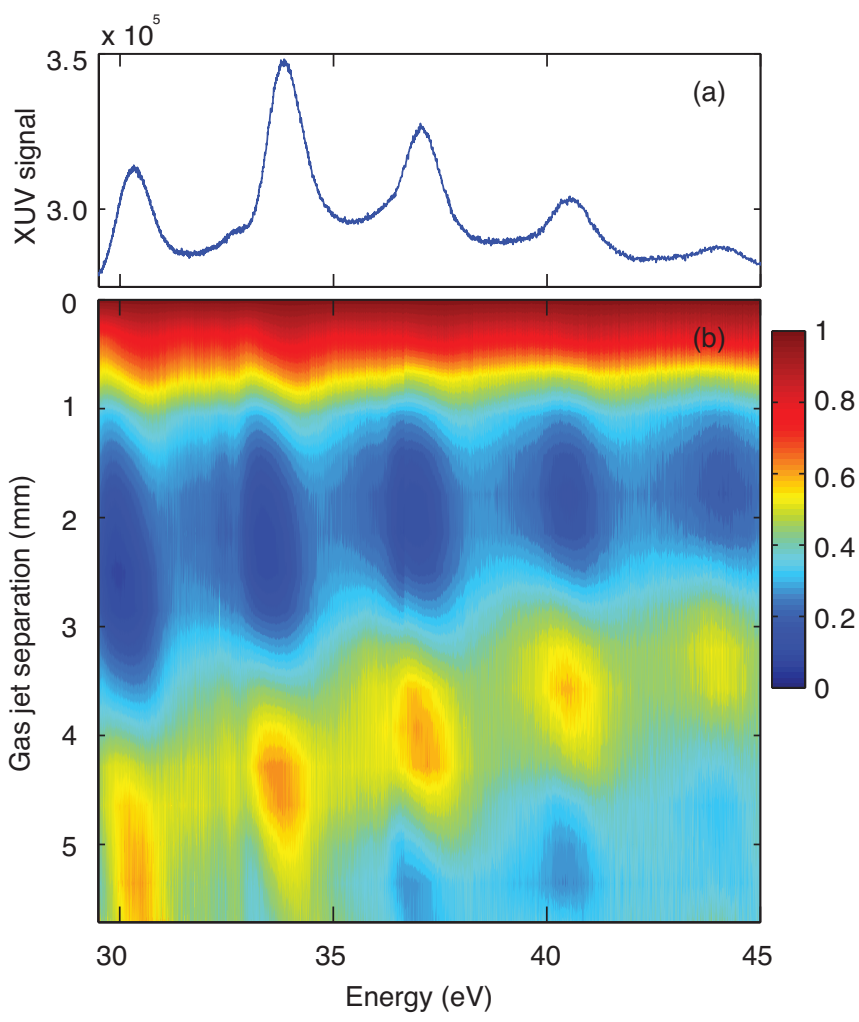

FIG. 2. A reference spectrum showing 5 of the harmonics captured (21st to the 29th) when only gas jet 2 was producing harmonics is given in (a). (b) depicts the normalised yield of the spectrum as the gas jet separation is increased. A normalised yield of 1 means that the HHG radiation being generated from each medium is perfectly in-phase whereas a normalised yield of 0 refers to the radiation being exactly out-of-phase. It can be seen that destructive interference first occurs for each harmonic, followed by constructive interference at a later separation. A second destructive interference is then observed for the harmonics beyond $35 \mathrm{eV}$. The gas jet separation cannot be increased beyond $6 \mathrm{~mm}$ due to experiment constrictions.

To test the HHG interferometer experimentally, two thin gas jets (thin relative to the Rayleigh range of the driving laser) filled with argon gas at a backing pressure of 100 Torr were used as the generation media. This arrangement simplifies the experiment by being in the single-atom response regime, where phase-matching effects do not play a significant role. A commercial Femtolaser Femtopower Compact Pro that produces sub-5.6 fs pulses centered at $800 \mathrm{~nm}$ at a repetition rate of $1 \mathrm{kHz}$ and energy of $200 \mu \mathrm{J}$ was used to provide the driving laser for the HHG process. The pulses are focused by an off-axis parabolic mirror $(f=750 \mathrm{~mm})$ into a vacuum chamber that houses the dual gas-jets.
The gas jets emerge from $250 \mu \mathrm{m}$ diameter hypodermic syringes with tips located $<100 \mu \mathrm{m}$ from the laser beam. Gas jet 2 is statically located at the centre of the laser focus while jet 1 is scanned from the laser focus to before the focus (i.e $z<0$ ). A $200 \mathrm{~nm} \mathrm{Al}$ foil is used as a filter to block the fundamental IR beam. The XUV spectrum in the 30 to $75 \mathrm{eV}$ range was measured using a spectrograph consisting of a grazing-incidence grating spectrometer (model: Hettrick Scientific ES-EUV-K) and an XUV camera (model: PI Pixus-XO). The characterization of the Rayleigh range by the beam waist method is performed with a CCD beam profiler (model: Thor Labs BC106-Vis) mounted on a translation stage in the direction of the beam propagation. In our experiment, the Gouy phase shift has been retrieved using two separate methods; profiling the divergence (as in Fig. 1(a) and reconstructing the propagation from beam planes. Both methods are found to be consistent with one another and equation (1).

Fig. 2 shows the measurement of the normalised yield of a five harmonics between 30 and $45 \mathrm{eV}$ as gas jet 1 is moved back from the focus. This data has been normalised to the coherent sum of the intensities $\left(I_{\mathrm{N}}=I_{1}+I_{2}+2 \sqrt{I_{1} I_{2}}\right)$ originating from each gas jet individually $\left(I_{1}\right.$ and $\left.I_{2}\right)$. It can be clearly seen that each harmonic undergoes destructive and then constructive interference at a particular gas jet separation. One concern is that the emitted XUV from jet 1 has a substantially altered spatiotemporal profile to that from jet 2 due to the focus conditions being different. Observations of the combined XUV emission in the far field showed no spatial interference effects, indicating that the spatial wavefunction of XUV emission was the same for each jet. The CEP of the laser was not locked for this measurement, effectively causing it to be randomised between any driving pulses. Further analysis of this data set can be used to test the original hypothesis that the Gouy phase is responsible for imparting a relative time delay between the emissions from the two gas jets. By finding the separation when a harmonic has undergone a $2 \pi$ phase shift (the coherent revival distance $z_{\mathrm{c}}$ ), such that the normalised yield approaches a peak, Eq. (5) can be solved for this condition such that,

$$
z_{\mathrm{c}}=2 \pi\left[q \frac{d \phi_{\text {Gouy }}}{d z}+\frac{d \phi_{S}}{d z}\right]^{-1} .
$$

By plotting the coherent revival distance, $1 / z_{\mathrm{c}}$, versus harmonic order, $q$, and fitting Eq. (6), the derivative of the Gouy phase and of the action phase can be found. Fig. 3 depicts this graph along with two theory lines. The blue errorbars represent data that was found by finding the revival distance of many harmonics. The green line is on the graph to demonstrate how close the results come to agreeing with a purely Gouy phase shift, with the red line including the effect of 
the action term. From the fit, the Rayleigh range of the driving laser can be calculated and compared to the value determined by profiling the beam waist. The measured Rayleigh range was $10.9 \pm 0.5 \mathrm{~mm}$ and the value obtained from the curve fit is $12.4 \pm 2.3 \mathrm{~mm}$. Both of these values are in reasonable agreement with each other, indicating that the mechanism behind the phase delay is predominantly due to the Gouy phase shift of the driving laser introducing a relative time delay between the electron recombination in each gas jet. The value found for the derivative of the action phase from the curve fit was found to be $-78 \pm 62 \mathrm{rad} / \mathrm{m}$, which is statistically insignificant when compared with the Gouy phase contribution.

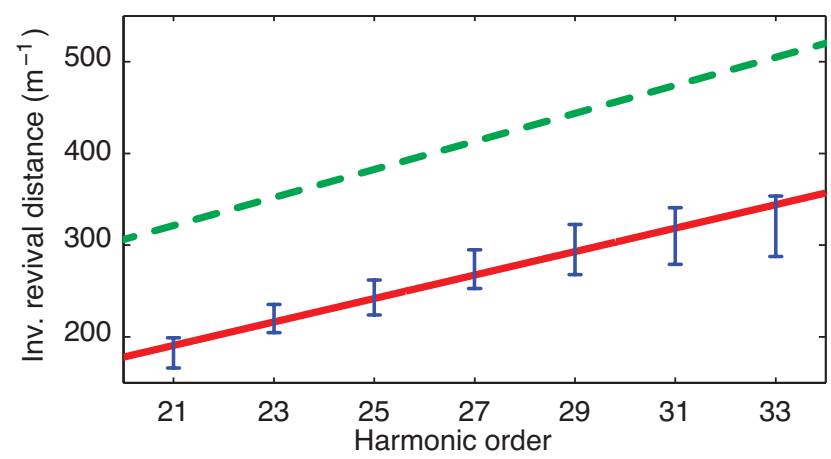

FIG. 3. The position at which the HHG radiation was found to constructively interfere (called the revival distance, here) was determined from the experimental data. Plotted here are the inverse of these positions versus the harmonic order of interest (blue errorbars are data). The green-dashed line represents the theoretical prediction of the inverse revival distance if only the Gouy phase were wholly responsible. The red-solid line is a curve fit to the data using Eq. (6) with the derivative of the Gouy and action phases as fitting parameters. From the Gouy fitted parameter a Rayleigh range of $12.4 \pm 2.3 \mathrm{~mm}$ was obtained which compares very will with the measured Rayleigh range of $10.9 \pm 0.5 \mathrm{~mm}$. The derivative of the action phase was found to be statistically insignificant at $-78 \pm 62 \mathrm{rad} / \mathrm{m}$, showing that the Gouy phase is the predominant effect in delaying the electron recombination and, hence, the photon emission time.

The interferometer has been demonstrated to provide a temporal delay between coherent XUV pulses generated by the HHG process and now, the ultimate timing resolution can be investigated. To accomplish this, the gas jet separation was set to where the normalised yield was approximately 0.5 for the harmonic at $30.5 \mathrm{eV}$ and the intensity of the harmonic was observed at $130 \mathrm{~ms}$ intervals for a period of one hour. The intensity data was then converted to a series of time delays by using a fit to the yield of a harmonic vs the gas jet separation. An Allan deviation on the time series can be performed as it characterises the uncertainty in a quantity being measured as a function of experimental measurement time [18]. Essentially, it is a tool that allows for an experimentalist to choose an amount of time that gives an appropriate uncertainty in the resultant measurement. This makes it ideal for characterising the ultimate timing resolution of the HHG interferometer. The Allan deviation is given by:

$$
\sigma_{\text {Allan }}(\tau)=\left(\frac{1}{2(N-2)} \sum_{i=1}^{N-2}\left(y_{i+1}-y_{i}\right)^{2}\right)^{1 / 2}
$$

where $\tau$ is the measurement time, $N$ is the number of samples and $y_{i}$ is the $i$ th sample in the time series. As shown in figure 4 , the interferometer achieves better than $100 \mathrm{zs}$ timing resolution for averaging times between 8 and $30 \mathrm{~s}$. This time frame is governed by the stability of the few-cycle laser system. The purely passive HHG interferometer achieves two orders of magnitude better timing resolution then the previous best value provided by an interferometer of 20 as, which required active stabilisation [5]. Recent experiments utilising active pulse shaping techniques with feedback stabilisation have demonstrated the ability to precisely delay two $10 \mathrm{fs}, 800 \mathrm{~nm}$ pulses down to $280 \mathrm{zs}[19,20]$. However, these experiments were using infrared wavelengths and is significantly easier to implement with optics than for extreme ultraviolet wavelengths where high-reflective optics are uncommon.

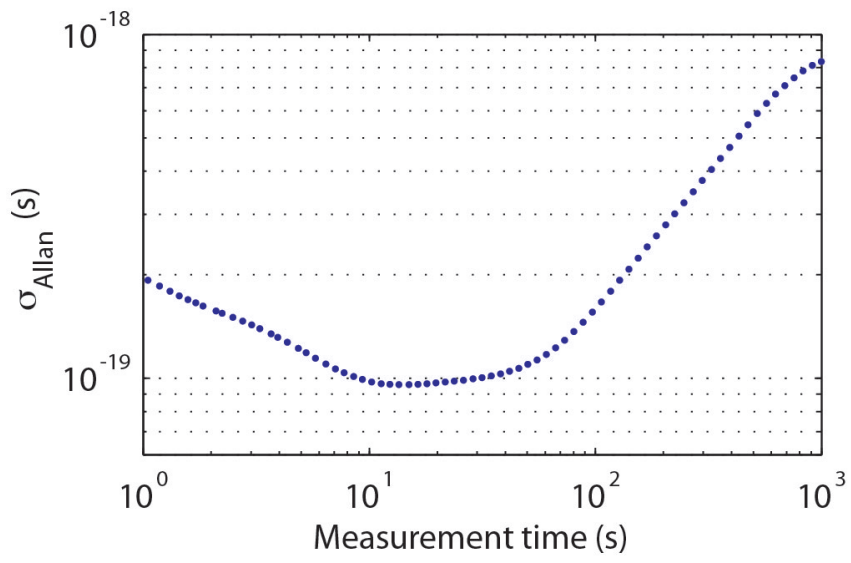

FIG. 4. The Allan deviation of timing resolution for the Gouy phase interferometer. The timing stability is better than $100 \mathrm{zs}$ for averaging times between 8 and 30 seconds.

In conclusion, the HHG interferometer allows for multiple XUV pulses to be emitted with a highly-controllable time delay by varying the position of generating media in a laser focus. It has been shown that the Gouy phase shift is the predominant effect responsible for imparting the relative time delay. The other effect to have some influence in the determining the phase shift comes from 
the phase the electron wavepacket accumulates when it is travelling in the electric-field. This "action phase" can be approximated by a first order Taylor expansion and was found to be statistically insignificant when compared to the contribution of the Gouy phases. The HHG interferometer provides an unprecedented level of timing control, down to better than $100 \mathrm{zs}$, when working with XUV, providing an improvement of two orders of magnitude over previous techniques. Applications for the HHG interferometer will include generating appropriately timed radiation for XUV pump-XUV probe attosecond dynamic experiments, pulse-shaping of XUV pulses and to directly compare atomic dipole effects in different atomic species by using different gases.

This research was funded by Australian Research Council grant DP0878560 and the Australian Research Council's Centre of Excellence in Coherent X-Ray Science. DEL, WCW and MGP were supported by Australian Postgraduate Awards.

* r.sang@griffith.edu.au

[1] Corkum, P. \& Krausz, F. Attosecond science. Nature Physics 3, 381-387 (2007).

[2] Krausz, F. \& Ivanov, M. Attosecond physics. Reviews of Modern Physics 81, 163-234 (2009).

[3] Wörner, H., Bertrand, J., Kartashov, D., Corkum, P. \& Villeneuve, D. Following a chemical reaction using highharmonic interferometry. Nature 466, 604-607 (2010).

[4] Goulielmakis, E. et al. Real-time observation of valence electron motion. Nature 466, 739-743 (2010).

[5] Chini, M. et al. Delay control in attosecond pump-probe experiments. Optics Express 17, 21459-21464 (2009).

[6] Seres, J. et al. Coherent superpostion of laser-driven soft$\mathrm{X}$-ray harmonics from successive sources. Nature Physics 3, 878-883 (2007).

[7] Chang, Z. \& Corkum, P. Attosecond photon sources: The first decade and beyond. Journal of the Optical Society of America B: Optical Physics 27, B9-B17 (2010).

[8] Gilbertson, S. et al. Isolated attosecond pulse generation using multicycle pulses directly from a laser amplifier. Physical Review A - Atomic, Molecular, and Optical
Physics 81, 043810 (2010).

[9] Hentschel, M. et al. Attosecond metrology. Nature 414, 509-513 (2001).

[10] Goulielmakis, E. et al. Single-cycle nonlinear optics. Science 320, 1614-1617 (2008).

[11] Corkum, P. Plasma perspective on strong field multiphoton ionization. Physical Review Letters 71, 1994-1997 (1993).

[12] Nisoli, M. et al. Effects of carrier-envelope phase differences of few-optical-cycle light pulses in single-shot high-order-harmonic spectra. Physical Review Letters 91, 213905 (2003).

[13] Lindner, F. et al. Gouy phase shift for few-cycle laser pulses. Physical Review Letters 92, 113001 (2004).

[14] Gouy, L.G. Sur une propriete nouvelle des ondes lumineuses. C. R. Acad. Sci. Paris 110, 1251 (1890).

[15] Nugent, K.A. X-ray image reconstruction using the transport of intensity equation. Proc. 8th Int. Conf. X-ray Microscopy IPAP Conf. Series 7, 399-402 (2005).

[16] Porras, M. Characterization of the electric field of focused pulsed gaussian beams for phase-sensitive interactions with matter. Optics Letters 34, 1546-1548 (2009).

[17] Spielmann, C. et al. Near-keV coherent X-ray generation with sub-10-fs lasers. IEEE Journal on Selected Topics in Quantum Electronics 4, 249-264 (1998).

[18] Allan, D. Statistics of atomic frequency standards. Proceedings of the IEEE 54, 221-230 (1966).

[19] Köhler, J. et al. Zeptosecond precision pulse shaping. Optics Express 19, 11638-11653 (2011).

[20] Horiuchi, N. Zeptosecond accuracy. Nature Photonics 5, 513 (2011)

[21] Baltuška, A. et al. Attosecond control of electronic processes by intense light fields. Nature 421, 611-615 (2003).

[22] Bandrauk, A., Chelkowski, S. \& Nguyen, H. Attosecond localization of electrons in molecules. International Journal of Quantum Chemistry, 100, 834-844 (2004).

[23] Hu, S. \& Collins, L. Attosecond pump probe: Exploring ultrafast electron motion inside an atom. Physical Review Letters 96, 1-4 (2006).

[24] Balcou, P., Salieres, P., \& L'Huillier, A. Generalized phase-matching conditions for high harmonics: The role of field-gradient forces. Physical Review A 55, 3204-3210 (1997).

[25] Lewenstein, M., Salires, P., \& L'Huillier, A. Phase of the atomic polarization in high-order harmonic generation. Physical Review A 52, 4747-4754 (1995). 